\title{
Wave of Chaos and Pattern Formation in Spatial Predator-Prey Systems with Holling Type IV Predator Response
}

\author{
R. K. Upadhyay ${ }^{a 1}$, N. Kumari ${ }^{a 2}$ and V. Rai ${ }^{b}$ \\ ${ }^{a}$ Department of Applied Mathematics, \\ Indian School of Mines University, Dhanbad 826 004, \\ Jharkhand, India. \\ ${ }^{b}$ Centre for Biomedical Engineering, Indian Institute of Technology New Delhi, \\ Hauz Khas, Delhi 110 016, India.
}

\begin{abstract}
The challenges to live in the open water and the diversity of habitats in the marine environments prompts phytoplankton to devise strategies which often involve production of toxins by Harmful Algal Bloom (HAB) and rapid production of metabolites from non-toxic precursor. The functional response of the predator is described by Holling type IV. We investigate wave phenomena and non-linear non-equilibrium pattern formation in a phytoplankton-zooplankton system with Holling type IV functional response. Type IV functional response yields to type II response in the event of large immunity from or tolerance of prey. It has been found that the Wave of Chaos (WoC) is still effective mechanism for the propagation of chaotic dynamics in predation and competitive systems. Fish predation has a significant role in the temporal evolution of spatial patterns of phytoplankton-zooplankton system. From a field ecologist's perspective, it is not only important to know the stable stationary patterns of an evolving phytoplankton-zooplankton system, but also to know at what point of time these observations are carried out. This has implications for ecological theory.
\end{abstract}

Key words: spatial predator-prey system, hopf-bifurcation, reaction-diffusion, pattern formation. AMS subject classification: 35K57, 37D45

\footnotetext{
${ }^{1}$ Corresponding author. E-mail: ranjit.upadhyay@yahoo.com

${ }^{2}$ Presently at Department of Mathematics, S.S.L.N.T. Women's College, Dhanbad, Jharkhand - 826001
} 


\section{Introduction}

Incorporation of spatial structure is crucial to ecological modeling and theory. This has not been done successfully in remote past because of problems related to computing power. As the computer technology is advancing, so is the spatial ecology. For example Allen et al. [3] have demonstrated that Nicholson-Bailey host-parasitoid system can be prevented from extinction by a spatial 'rescue effect' even in a uniform spatially explicit environment if dispersal is made small enough relative to the size of spatial domain. Pascual [31] observed diffusion induced chaos in a spatial prey-predator system and its robustness was studied by Rai and Jayaraman [37]. Any dynamical behavior is considered to be robust (i.e., likelihood of observation is maximized) when it is detected in a dense set formed by key parameters of dynamical systems. Rai and Jayaraman [37] studied deterministic chaos in two spatial predator prey system with a view to examine if diffusion-induced chaos is robust? The chaotic behavior in these systems is generated when stable limit cycle oscillators of comparable frequencies couple together to force each other. The origin of these oscillators takes place on a spatial gradient of the specific growth rate of the prey. Such gradients occur in natural aquatic environments because of spatial distribution of the nutrients. How patchiness is generated and maintained in natural ecologies has been an intense subject of research since early nineties (see [21, 22]), but its effect on ecological dynamics could be modeled and studied only recently. Four main methods exist now to study the influence of spatial structure on ecological dynamics: i) reaction-diffusion systems, ii) coupled map lattices, iii) cellular automata, and iv) integro-difference equations. In this paper, we choose to work with reaction-diffusion systems.

Among ecology's oldest concerns is development and maintenance of spatio-temporal patterns (see $[21,26]$ ). Investigation of consequences of these patterns for dynamics and functioning of ecosystems is a task at the heart of ecology. The dynamics of interacting populations in connection to spatial phenomena such as pattern formation and spatiotemporal chaos have recently become a focus of intensive research in theoretical ecology. Liu et al. [23] studied a spatial phytoplanktonzooplankton system with periodic forcing and additive noise. Following these models they considered, the Holling type IV predator-prey model with external periodic forcing and colored noise. Zhang et al. [47] demonstrate that two species spatial systems could be useful to explain spatiotemporal behavior of populations whose dynamics is strongly affected by noise and the environmental physical variables.

The challenges in spatial ecology relate to modeling and analysis of asymmetric dispersal which results from multiple selective pressures. Population kin structure is one of the factors causing selective pressure [13]. However, in the present paper, we assume that both species perform local random movements. These local movements can be modeled by Fickian diffusion terms and results in reaction diffusion (R-D) system which can easily be studied using computer simulations. The conceptual applicability of diffusion terms to describe re-distribution of species in space due to random motion of the individuals for any value of population density was shown by Okubo [29]. Since the dispersal rates are assumed to be the same for prey and predator, the patterns cannot appear due to the Turing instability [38] and should be ascribed to another mechanism [33]. Depending on the details of the species distribution, there can be two different patterns corresponding to two different regimes of the system dynamics i.e., regular or chaotic [35]. The objective of the 
present work is to examine how functional responses of predators influence pattern formation in R-D systems containing same basic ingredients.

Holling type IV functional response is relatively less studied in the population ecology. This response function describes a situation in which the predator's per capita rate of predation decreases at sufficiently high prey densities due to either prey interference or prey toxicity. The prey toxicity results from either environmental contaminants or poisonous substances released by phytoplankton under increased predation pressure [9, 12, 17]; e.g., cynobacterium Anabaena affinis. Upadhyay and Chattopadhyay [43] studied the model in which extra mortality term for zooplankton is considered due to the toxin liberation process of phytotoplankton.

This paper aims to investigate if functional responses influence the spatial patterns in aquatic systems (marine or oceanic). We study two reaction-diffusion systems with and without fish predation. The paper is organized as follows. We describe model systems in the second section which were thoroughly analyzed for WoC. The stability and bifurcation analysis of the model systems are presented in third section. In the fourth section, conditions for diffusion driven instability and wave number for model system 2 is obtained. The results of simulation experiments are presented in the fifth section. The sixth section summarizes main results of the present study. It also discusses implications of these results for harvesting of natural resources in marine and oceanic systems.

\section{The model systems}

In this section, we consider two predator-prey systems with Holling type IV functional response. Holling type IV functional response was first introduced by Haldane [16] in enzymology. Some experiments indicate that the non monotonic response occurs at the microbial level: when the nutrient concentration reaches a high level an inhibitory effect on the specific growth rate may occur $[5,8,11,45]$. This is often seen when microorganisms are used for waste decomposition or for water purification [14]. Such an inhibitory effect is modeled by a function called the MonodHaldane function [30], which is similar to the Monod (i.e., the Michaelis-Menten) function for low concentrations but includes the inhibitory effect at high concentrations. Collings [10] also used the response function in a mite predator-prey interaction model and called it a Holling typeIV function. The function is also used for the dependence of the growth rate on an inhibitory substrate [11]. For large predator's immunity, Holling type IV functional response reduces to Holling type II [20]. Over a period of time, predators evolve tolerance of and immunity from the prey toxicity. The effect of this evolutionary process on the per capita predation rate of the prey has been included in Type IV non-monotone functional responses [14] by introducing the parameter $i$ in the denominator of the quadratic term. Co-evolution of predator and prey in ecological systems (aquatic or terrestrial) guarantee predator's immunity.

\subsection{Model system 1}

We first consider the predator-prey model system of the form: 


$$
\begin{aligned}
& \frac{\partial P}{\partial T}=\frac{\xi N P}{H_{N}+N}-\phi P^{2}-\frac{c P Z}{\frac{P^{2}}{i}+P+a}+D_{P} \Delta P \\
& \frac{\partial Z}{\partial T}=\frac{b c P Z}{\frac{P^{2}}{i}+P+a}-m Z+D_{Z} \Delta Z
\end{aligned}
$$

where $P$ and $Z$ are functions of time and space representing population densities of prey and predator respectively. All the parameters assume non-negative values. $\xi$ is the maximum per capita growth rate of prey. $\phi$ is the intensity of competition among individuals of prey population. $c$ is the rate at which prey is grazed which follows Holling type IV functional response. $b$ is the conversion coefficient from individuals of prey into individuals of predator. $m$ is the mortality rate of predator. $D_{P}$ and $D_{Z}$ are the diffusion coefficients of prey and predator respectively. $i$ is a direct measure of the predator's immunity from or tolerance of the prey. Predator's foraging efficiency decreases with decrease in parameter $i$. At high prey densities, the zooplankton's per capita grazing rate decreases with density because of inhibitory effects. It is not clear how these inhibitory effects are caused. $a$ is the half-saturation constant in the absence of any inhibitory effect. $N$ is the food for prey and $H_{N}$ is the prey density at which specific growth rate becomes half its saturation value.

\subsection{Model system 2}

The second predator-prey model system is:

$$
\begin{aligned}
& \frac{\partial P}{\partial T}=\frac{\xi N P}{H_{N}+N}-\phi P^{2}-\frac{c P Z}{\frac{P^{2}}{i}+P+a}+D_{P} \Delta P \\
& \frac{\partial Z}{\partial T}=\frac{b c P Z}{\frac{P^{2}}{i}+P+a}-m Z-\frac{F Z^{2}}{H_{Z}^{2}+Z^{2}}+D_{Z} \Delta Z
\end{aligned}
$$

where $F$ is the predation rate of predator population, which follows Holling type III functional response. The other parameters are the same as explained for model system 1 .

For simplification, we define following parameters in both the model systems

$$
\frac{\xi N}{H_{N}+N}=r, \quad \frac{r}{\phi}=l .
$$

The model system 1 in dimensionless form can be written as

$$
\begin{aligned}
\frac{\partial u}{\partial t} & =u\left(1-\frac{u}{K}\right)-\frac{u v}{\frac{u^{2}}{\alpha}+u+1}+\Delta u \\
\frac{\partial v}{\partial t} & =\frac{\beta u v}{\frac{u^{2}}{\alpha}+u+1}-\gamma v+D \Delta v
\end{aligned}
$$


where the dimensionless quantities are

$$
u=\frac{P}{a}, \quad v=\frac{c Z}{a r}, \quad x_{i}=X_{i}\left[\frac{r}{D_{P}}\right]^{1 / 2}, \quad t=r T,
$$

and new parameters take the form

$$
K=\frac{l}{a}, \quad \alpha=\frac{i}{a}, \quad \beta=\frac{b c}{r}, \quad \gamma=\frac{m}{r}, \quad D=\frac{D_{Z}}{D_{P}} .
$$

Dimensionless form of the model system 2 is

$$
\begin{aligned}
& \frac{\partial u}{\partial t}=u\left(1-\frac{u}{K}\right)-\frac{u v}{\frac{u^{2}}{\alpha}+u+1}+\Delta u, \\
& \frac{\partial v}{\partial t}=\frac{\beta u v}{\frac{u^{2}}{\alpha}+u+1}-\gamma v-\frac{f v^{2}}{v^{2}+\eta^{2}}+D \Delta v,
\end{aligned}
$$

where the new parameters are

$$
f=\frac{F c}{a r^{2}}, \quad \eta=\frac{c H_{Z}}{a r}
$$

\section{Stability and bifurcation analysis}

In this section, we restrict ourselves to the detailed stability analysis of the models in the absence of diffusion in which only the interaction part of the model system 1 and model system 2 are taken into account. We find the non-negative equilibrium states of both the models and discuss their stability properties with respect to variation of several parameters. We also perform Hopfbifurcation analysis of the model systems. The steady states loose stability in a Hopf-Bifurcation and it leads to the emergence of periodic behaviour.

\subsection{Analysis of model system 1}

The model system 1 in the absence of diffusion can be written as:

$$
\begin{aligned}
\frac{d u}{d t} & =u\left(1-\frac{u}{K}\right)-\frac{u v}{\frac{u^{2}}{\alpha}+u+1}, \\
\frac{d v}{d t} & =\frac{\beta u v}{\frac{u^{2}}{\alpha}+u+1}-\gamma v .
\end{aligned}
$$

It is readily seen [7] that the system (3.5) possesses four non-negative equilibria namely $E_{0}=$ $(0,0), E_{1}=(K, 0)$ and $E_{ \pm}^{*}=\left(u^{*}, v^{*}\right) . E_{ \pm}^{*}$ are the non-negative nontrivial points of coexistence of both prey and predator. For non-trivial points $E_{ \pm}^{*}\left(u^{*}, v^{*}\right)$

$$
u^{*}=\frac{-S \pm \sqrt{S^{2}-4 \alpha}}{2}, \quad v^{*}=\left(1-\frac{u^{*}}{K}\right)\left(\frac{u^{* 2}}{\alpha}+u^{*}+1\right),
$$


where $S=\alpha\left(1-\frac{\beta}{\gamma}\right)$.

The non-trivial points $E_{ \pm}^{*}$ exist in the biologically meaningful domain $u \geq 0, v \geq 0$ under the restraints

$$
\left(1-\frac{\beta}{\gamma}\right)^{2}>\frac{4}{\alpha} \quad \text { and } \quad u^{*}<K
$$

Now we study the stability criteria of different equilibrium points of the model system. $E_{0}$ is a saddle point with an unstable manifold along $u$-direction and stable manifold along $v$-direction. $E_{1}$ is locally asymptotically stable in $u v$ plane provided the inequality $\frac{\beta K}{\frac{K^{2}}{\alpha}+K+1}<\gamma$ is satisfied. If $\frac{\beta K}{\frac{K^{2}}{\alpha}+K+1}>\gamma$, then the equilibrium point $E_{1}$ is a saddle point with stable manifold locally in the $u$-direction and with unstable manifold locally in the $v$-direction or a saddle node if $\frac{\beta K}{\frac{K^{2}}{\alpha}+K+1}=\gamma$.

In the following theorem we are able to find necessary and sufficient conditions for non trivial positive equilibrium points $E_{ \pm}^{*}$ to be locally asymptotically stable. The proof of this theorem follows from the Routh-Hurwitz criteria, and hence it is omitted. For this purpose we use the following notations:

$$
\begin{aligned}
& A=\frac{u^{*}}{K}\left[1-\frac{K v^{*}\left(\alpha+2 u^{*}\right)}{\alpha\left(\frac{u^{* 2}}{\alpha}+u^{*}+1\right)^{2}}\right], \\
& B=\frac{\beta u^{*} v^{*}\left(1-\frac{u^{* 2}}{\alpha}\right)}{\left(\frac{u^{* 2}}{\alpha}+u^{*}+1\right)^{3}} .
\end{aligned}
$$

Theorem 3.1 The non-trivial positive equilibrium points $E_{ \pm}^{*}$ are locally asymptotically stable provided the following conditions are satisfied:

$$
\alpha\left(\frac{u^{* 2}}{\alpha}+u^{*}+1\right)^{2}>K\left(\alpha+2 u^{*}\right) v^{*} \text { and } u^{* 2}<\alpha,
$$

Remark 3.2 The conditions in eq. (3.8) are obtained if the inequalities $A>0$ and $B>0$ hold.

For bifurcation analysis, we consider the variational matrix at $E_{ \pm}^{*}$ of the form,

$$
V^{*}=\left[\begin{array}{cc}
\frac{\gamma}{\beta} q^{\prime}\left(u^{*}\right) & -\frac{\gamma}{\beta} \\
\gamma w^{\prime}\left(u^{*}\right) q\left(u^{*}\right) & 0
\end{array}\right],
$$

where $q(u)=\left(1-\frac{u}{K}\right)\left(\frac{u^{2}}{\alpha}+u+1\right), w(u)=\frac{\beta}{\gamma} p(u)-1$ and $p(u)=\frac{u}{\frac{u^{2}}{\alpha}+u+1}$ are obtained by writing (3.5) in the form of the functions of $u$. Hence, the corresponding characteristic equation will be

$$
\lambda^{2}-\frac{\gamma}{\beta} q^{\prime}\left(u^{*}\right) \lambda+\frac{\gamma^{2}}{\beta} w^{\prime}\left(u^{*}\right) q\left(u^{*}\right)=0 .
$$

For the non-trivial point $E_{+}^{*}, u^{*}=\frac{-S+\sqrt{S^{2}-4 \alpha}}{2}$, therefore in eq.(3.9), $w^{\prime}\left(u^{*}\right)<0$ and $q\left(u^{*}\right)>0$. Descarte's rule of signs implies that the two eigenvalues are real and of opposite sign, regardless of the sign of $q^{\prime}\left(u^{*}\right)$. The equilibrium point $E_{+}^{*}$ is an unstable saddle point. 
For the non-trivial point $E_{-}^{*}$, we consider $u^{*}=\frac{-S-\sqrt{S^{2}-4 \alpha}}{2}$. But $w^{\prime}\left(u^{*}\right)>0$ and $q\left(u^{*}\right)>0$, from the conditions of existence of $E_{-}^{*}$. Therefore by Routh-Hurwitz criteria, $E_{-}^{*}$ is asymptotically stable if $q^{\prime}\left(u^{*}\right)<0$ and unstable if $q^{\prime}\left(u^{*}\right)>0$. Therefore $q^{\prime}\left(u^{*}\right)=0$ is a bifurcation point. But $q^{\prime}\left(u^{*}\right)=0$ gives,

$$
K=\frac{\frac{3 u^{* 2}}{\alpha}+2 u^{*}+1}{1+\frac{2 u^{*}}{\alpha}}=\frac{-11+\alpha(6 s-1)\left(s+\sqrt{s^{2}-\frac{4}{\alpha}}\right)}{-s-\sqrt{s^{2}-\frac{4}{\alpha}}} \equiv \bar{K}
$$

where $s=\frac{S}{\alpha}=\left(1-\frac{\beta}{\gamma}\right)$. From eq. (3.10), by applying Hopf-bifurcation theorem, it can be shown that a limit cycle exists near $q^{\prime}\left(u^{*}\right)=0$.

Theorem 3.3 For the equilibrium point $E_{-}^{*}\left(u^{*}, v^{*}\right)$, a Hopf-bifurcation occurs at the parameter value $K=\bar{K}$ given in eq. (3.10).

From the above stability analysis we can conclude that, the point of extinction of both prey and predator i.e., $E_{0}$ is always unstable. The equilibrium point $E_{1}$, which corresponds to the existence of prey at its carrying capacity and extinction of the predator, is stable or unstable depending upon the parameter values. The non-trivial point $E_{-}^{*}$ of prey-predator coexistence will undergo a Hopfbifurcation as the carrying capacity of the prey $K$ attains a constant value $\bar{K}$.

Kolmogorov theorem $[18,27]$ is mathematically robust and is widely applicable to predatorprey models used in theoretical biology. The amplitude and the period of stable limit cycle oscillations depend on the intrinsic attributes of the systems i.e. system parameters. It is used to obtain conditions on system parameters which lead us to the period-doubling route to chaos and is based on the Poincare-Benedixon theory [27]. The theorem guarantees the existence of stable equilibrium points or limit cycles in two-dimensional nonlinear systems provided they fulfill certain requirements. Kolmogorov theorem strongly suggests that those natural ecosystems which seem to exhibit a persistent pattern of reasonably regular oscillations are in fact stable limit cycles [19].

An application of Kolmogorov theorem (see Appendix) to model system 1 without diffusion (system (3.5)) yields the following conditions:

$$
\left(1-\frac{\beta}{\gamma}\right)^{2}>\frac{4}{\alpha} \text { and } \beta>\gamma .
$$

A close examination of Kolmogorov inequalities results in the conclusion that the only meaningful inequality is expressed by eq.(3.11). Figure 1 shows the area which houses stable limit cycle solutions. The parameter values for simulation experiments are derived from the shaded area as the wave phenomena supported by the model systems presented in the previous section is critically dependent on oscillatory dynamics of the interaction part of the model system. 


\subsection{Analysis of model system 2}

The model system 2 in the absence of diffusion takes the form as under:

$$
\begin{aligned}
& \frac{d u}{d t}=u\left(1-\frac{u}{K}\right)-\frac{u v}{\frac{u^{2}}{\alpha}+u+1}, \\
& \frac{d v}{d t}=\frac{\beta u v}{\frac{u^{2}}{\alpha}+u+1}-\gamma v-\frac{f v^{2}}{v^{2}+\eta^{2}} .
\end{aligned}
$$

An application of linear stability analysis to the model gives three stationary points of model system 2. The equilibrium points of model system (3.12) can be obtained by solving $d u / d t=0$, $d v / d t=0$. It can be seen that model system 2 has three nonnegative equilibria, namely, $E_{0}=$ $(0,0), E_{1}=(K, 0)$ and $E^{*}=\left(u^{*}, v^{*}\right)$. The other two equilibrium points are imaginary which are of no biological interest because the predation rate of zooplankton by fish population is always assumed to be non-negative.

The first two equilibria $E_{0}$ and $E_{1}$ obviously exist. The existence of the positive equilibrium $E^{*}$ can be shown as follows.

It may be noted that $u^{*}$ and $v^{*}$ are the positive solutions of the following algebraic equations:

$$
\begin{array}{r}
v=\left(1-\frac{u}{K}\right)\left(\frac{u^{2}}{\alpha}+u+1\right), \\
\frac{f v}{v^{2}+\eta^{2}}=\frac{\beta u}{\frac{u^{2}}{\alpha}+u+1}-\gamma .
\end{array}
$$

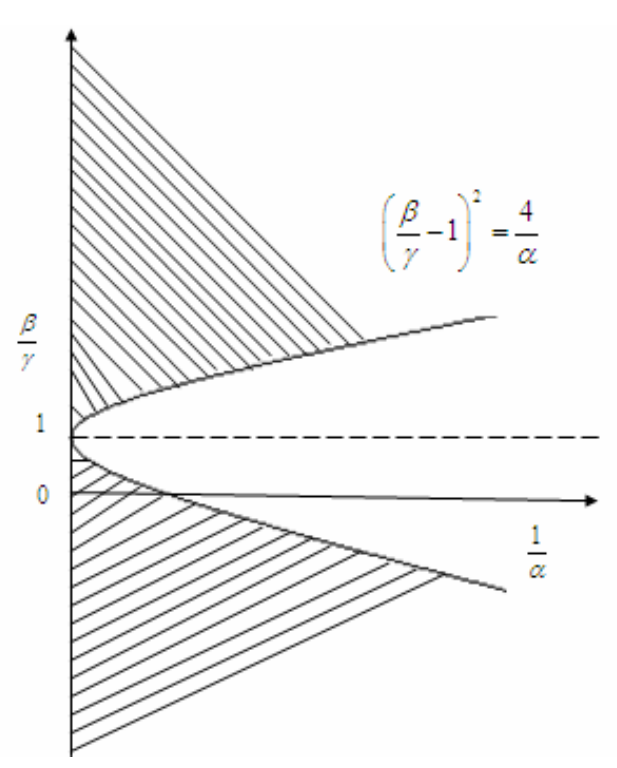

Figure 1: Shaded region houses stable limit cycle solutions. 
From first equation of (3.13) we note the following:

When $v=0$, then $u=K$, as $\left(\frac{u^{2}}{\alpha}+u+1\right)>0$.

When $u=0$, then $v=1$.

$$
\frac{d v}{d u}=-3 u^{2}+2(K-\alpha) u+(K-1) \alpha>0, \quad \text { if } \quad 2(K-\alpha) u+(K-1) \alpha>3 u^{2} .
$$

From second equation of (3.13) we note the following:

When $u=0$, then $v<0$.

When $v=0$, then $u=\frac{\alpha}{2}\left[\left(\frac{\beta}{\gamma}-1\right) \pm \sqrt{\left(1-\frac{\beta}{\gamma}\right)^{2}-\frac{4}{\alpha}}\right]>0$, is always true.

$$
\frac{d v}{d u}=\frac{\left(v^{2}+\eta^{2}\right)\left(\beta+\frac{2 \gamma u}{\alpha}+\gamma\right)-f v\left(\frac{2 u}{\alpha}+1\right)}{(f+2 \gamma v)\left(\frac{u^{2}}{\alpha}+u+1\right)-2 \beta u v} .
$$

It is clear from the above equation that $\frac{d v}{d u}$ is positive if

$$
\begin{aligned}
& \text { (i) }\left(v^{2}+\eta^{2}\right)\left(\beta+\frac{2 \gamma u}{\alpha}+\gamma\right)>f v\left(\frac{2 u}{\alpha}+1\right), \text { and } \\
& \text { (ii) }(f+2 \gamma v)\left(\frac{u^{2}}{\alpha}+u+1\right)>2 \beta u v .
\end{aligned}
$$

Remark 3.4 It may be noted that $\frac{d v}{d u}$ will remain positive even if both the inequalities in (3.16) are reversed.

From the above analysis we note that the two isoclines given in (3.13) intersect at a unique point $\left(u^{*}, v^{*}\right)$ in the positive quadrant of the $u v$-plane if, in addition to (3.16), condition (3.14) is also satisfied.

Thus, the positive equilibrium $E^{*}\left(u^{*}, v^{*}\right)$ exists uniquely if conditions (3.14) and (3.16) hold.

Now we study the nature and stability of each equilibrium points of the model system. To study the local stability behavior of the equilibria of model system 2 , we compute the variational matrices corresponding to each equilibrium point. From these matrices, following results are obtained.

$E_{0}$ is a saddle point with an unstable manifold along $u$-direction and stable manifold along $v$-direction. $E_{1}$ is locally asymptotically stable provided $\frac{\beta K}{\frac{K^{2}}{\alpha}+K+1}<\gamma$ otherwise unstable.

We propose the theorem for necessary and sufficient conditions of the positive equilibrium $E^{*}\left(u^{*}, v^{*}\right)$ to be locally asymptotically stable.

The proof of the theorem follows from the Routh-Hurwitz criteria, and hence omitted. We use the 
following notations:

Let us define the following:

$$
\begin{aligned}
& A_{1}=\frac{u^{*}}{K}\left(1-\frac{K v^{*}\left(\alpha+2 u^{*}\right)}{\alpha\left(\frac{u^{* 2}}{\alpha}+u^{*}+1\right)^{2}}\right)+\frac{f v^{*}\left(\eta^{2}-v^{* 2}\right)}{\left(\eta^{2}+v^{* 2}\right)^{2}} \\
& B_{1}=\frac{u^{*}}{K}\left(1-\frac{K v^{*}\left(\alpha+2 u^{*}\right)}{\alpha\left(\frac{u^{* 2}}{\alpha}+u^{*}+1\right)^{2}}\right)\left(\frac{f v^{*}\left(\eta^{2}-v^{* 2}\right)}{\left(\eta^{2}+v^{* 2}\right)^{2}}\right)+\frac{\beta u^{*} v^{*}\left(1-\frac{u^{* 2}}{\alpha}\right)}{\left(\frac{u^{* 2}}{\alpha}+u^{*}+1\right)^{3}} .
\end{aligned}
$$

Theorem 3.5 The unique non-trivial positive equilibrium point $E^{*}$ is locally asymptotically stable provided the following inequalities hold:

$$
\alpha\left(\frac{u^{* 2}}{\alpha}+u^{*}+1\right)^{2}>K\left(\alpha+2 u^{*}\right) v^{*}, \quad v^{* 2}<\eta^{2} \quad \text { and } \quad u^{* 2}<\alpha .
$$

Remark 3.6 Conditions of eq. (3.18) are obtained if the conditions $A_{1}>0$ and $B_{1}>0$ hold.

\section{Diffusion driven instability and wave number}

In this section, we study the effect of diffusion on the model systems about the interior equilibrium point. Instability will occur due to diffusion when a parameter varies slowly in such a way that a stability condition is suddenly violated and it can bring about a situation wherein perturbation of a non-zero (finite) wavelength starts growing (perturbations of zero wave number are stable when diffusive instability sets in). Turing instability cannot occur for the model system 1 because the equation for predator is linear with respect to predator population, $Z$ [38]. Therefore we present diffusion driven analysis of the complete model system 2 with initial condition

$$
u(x, 0)>0, \quad v(x, 0)>0, \quad \text { for } \quad x \in[0, R]
$$

and zero flux boundary conditions.

To study the effect of diffusion on the system, we have considered the linearized form of system (2.4) about $E^{*}\left(u^{*}, v^{*}\right)$ as follows:

$$
\begin{aligned}
& \frac{\partial U}{\partial t}=a_{11} U+a_{12} V+\frac{\partial^{2} U}{\partial x^{2}}, \\
& \frac{\partial V}{\partial t}=a_{21} U+a_{22} V+D \frac{\partial^{2} V}{\partial x^{2}},
\end{aligned}
$$


where $u=u^{*}+U, v=v^{*}+V \quad$ and

$$
\begin{array}{r}
a_{11}=1-\frac{2 u^{*}}{K}-\frac{v^{*}\left(1-\frac{u^{* 2}}{\alpha}\right)}{\left(\frac{u^{* 2}}{\alpha}+u^{*}+1\right)^{2}}, \quad a_{12}=\frac{-u^{*}}{\frac{u^{* 2}}{\alpha}+u^{*}+1}, \\
a_{21}=\frac{\beta v^{*}\left(1-\frac{u^{* 2}}{\alpha}\right)}{\left(\frac{u^{* 2}}{\alpha}+u^{*}+1\right)^{2}}, \quad a_{22}=\frac{f v^{*}\left(v^{* 2}-\eta^{2}\right)}{\left(v^{* 2}+\eta^{2}\right)^{2}} .
\end{array}
$$

It may be noted that $(U, V)$ are small perturbations of $(u, v)$ about the equilibrium point $\left(u^{*}, v^{*}\right)$. Let us assume the solutions of system of equations (4.20) of the form

$$
\left(\begin{array}{c}
U \\
V
\end{array}\right)=\left(\begin{array}{c}
a \\
b
\end{array}\right) \exp (\lambda t+i k x)
$$

where $\lambda$ and $k$ are the frequency and wave number respectively. The characteristic equation of the linearized system is given by

$$
\lambda^{2}+\rho_{1} \lambda+\rho_{2}=0
$$

where

$$
\begin{gathered}
\rho_{1}=A_{1}+(1+D) k^{2} \\
\rho_{2}=B_{1}+\left(\frac{f v^{*}\left(\eta^{2}-v^{* 2}\right)}{\left(\eta^{2}+v^{* 2}\right)^{2}}\right) k^{2}+\left(\frac{u^{*}}{K}-\frac{u^{*} v^{*}\left(\alpha+2 u^{*}\right)}{\alpha\left(\frac{u^{* 2}}{\alpha}+u^{*}+1\right)^{2}}+k^{2}\right) D k^{2}
\end{gathered}
$$

and $A_{1}, B_{1}$ are defined in (3.17).

From equations (4.22) and (4.23), and using the Routh-Hurwitz criteria, the following theorem follows immediately.

Theorem 4.1(i) The positive equilibrium $E^{*}$ is locally asymptotically stable in the presence of diffusion if and only if

$$
\rho_{1}>0 \text { and } \rho_{2}>0 .
$$

(ii) If the inequalities in eq. (3.18) are satisfied, then the positive equilibrium $E^{*}$ is locally asymptotically stable in the presence as well as absence of diffusion.

(iii) Suppose that any one or all of the inequalities in eq. (3.18) are not satisfied, i.e., either $A_{1}$ or $B_{1}$ is negative or both $A_{1}$ and $B_{1}$ are negative. Then by increasing $D$ to sufficiently large values, $\rho_{1}$ and $\rho_{2}$ can be made positive and hence $E^{*}$ can be made locally asymptotically stable.

Diffusive instability sets in when at least one of the conditions in eq. (4.24) is violated subject to the conditions $A_{1}>0$ and $B_{1}>0$ hold.

But it is evident that the first condition $\rho_{1}>0$ is not violated when the condition $A_{1}>0$ is met. Hence only the violation of condition $\rho_{2}>0$ gives rise to diffusive instability. Hence the condition for diffusive instability is given by 


$$
H\left(k^{2}\right)=D k^{4}+\left[D\left(\frac{u^{*}}{K}-\frac{u^{*} v^{*}\left(\alpha+2 u^{*}\right)}{\alpha\left(\frac{u^{* 2}}{\alpha}+u^{*}+1\right)^{2}}\right)+\frac{f v^{*}\left(\eta^{2}-v^{* 2}\right)}{\left(\eta^{2}+v^{* 2}\right)^{2}}\right] k^{2}+B_{1}<0 .
$$

$H$ is quadratic in $k^{2}$ and the graph of $H\left(k^{2}\right)=0$ is a parabola. The minimum of $H\left(k^{2}\right)=0$ occurs at $k^{2}=k_{c}^{2}$ where

$$
k_{c}^{2}=\frac{1}{2 D}\left[D\left(\frac{u^{*} v^{*}\left(\alpha+2 u^{*}\right)}{\alpha\left(\frac{u^{* 2}}{\alpha}+u^{*}+1\right)^{2}}-\frac{u^{*}}{K}\right)+\frac{f v^{*}\left(v^{* 2}-\eta^{2}\right)}{\left(v^{* 2}+\eta^{2}\right)^{2}}\right]>0,
$$

Consequently, the condition for diffusive instability is $H\left(k_{c}^{2}\right)<0$. Therefore

$$
\frac{1}{4 D}\left[D\left(\frac{u^{*} v^{*}\left(\alpha+2 u^{*}\right)}{\alpha\left(\frac{u^{* 2}}{\alpha}+u^{*}+1\right)^{2}}-\frac{u^{*}}{K}\right)+\frac{f v^{*}\left(v^{* 2}-\eta^{2}\right)}{\left(v^{* 2}+\eta^{2}\right)^{2}}\right]^{2}>B_{1}
$$

where $B_{1}$ is defined in (3.17).

Theorem 4.2(i) The final criterion for diffusive instability for the model system 2 is obtained by combining eqs. (4.26) and (4.27) leading to the following condition:

$$
\frac{D u^{*}}{K}\left(\frac{K v^{*}\left(\alpha+2 u^{*}\right)}{\alpha\left(\frac{u^{* 2}}{\alpha}+u^{*}+1\right)^{2}}-1\right)+\frac{f v^{*}\left(v^{* 2}-\eta^{2}\right)}{\left(v^{* 2}+\eta^{2}\right)^{2}}>2\left(B_{1} D\right)^{1 / 2}>0 .
$$

(ii) The critical wave number $k_{c}$ of the first perturbations is obtained by solving eq. (4.26).

For the model system $2, k_{-}<k_{c}<k_{+}$is a necessary condition to obtain Turing instabilities. In particular it gives no Turing instabilities conditions if $k_{-}>k_{c}$. Hence the condition obtained

$$
D<\frac{2 B_{1}}{a_{11}^{2}}-\frac{a_{22}}{a_{11}} \Longrightarrow \quad \text { No Turing instabilities, }
$$

where $a_{11}$ and $a_{22}$ are defined in eq. (4.21).

\section{Numerical simulations}

We have shown in the above section that the non-trivial homogeneous stationary state can loose its stability when diffusion is taken into consideration. Although this result seems absurd as diffusion process is known to have a stabilizing effect. However, Turing [42] observed that nonlinear chemical reactions can lead to an inhomogeneous distribution if diffusion is taken into account. These 
Turing instabilities, when excited by external perturbations of specific wavelengths, can appear within certain parameter spaces [4].

The dynamics of the model systems 1 and 2 are studied with the help of numerical simulation, both in one and two dimensions. To investigate the spatiotemporal dynamics of the model systems (2.3) and (2.4), we solved it numerically using semi-implicit (in time) finite difference method. The steps $\triangle x$ and $\triangle t$ of the numerical grid are chosen sufficiently small so that the results are independent of the step size. This method finally results to a sparse, banded linear system of algebraic equations. The linear system obtained, is solved by using LU factorization method for one-dimensional case and by GMRES algorithm [15] for two-dimensional case.

The plots (space vs. population densities) are obtained to study the spatial dynamics of the model systems. The temporal dynamics is studied by observing the effect of time on space vs. density plot of prey and predator populations. For two-dimensional case, the spatial snapshots of prey and predator densities are obtained by performing computer simulations at different time levels and we have tried to study the spatiotemporal dynamics of both the spatial model systems.

\subsection{The spatiotemporal dynamics in one dimensional case}

\subsubsection{Model system 1}

In this subsection, we perform a detailed study of the spatio-temporal dynamics of the nondimensionalized system (2.3) in one dimension. The model system with $u(x, t), v(x, t)$ and $\Delta=\frac{\partial^{2}}{\partial x^{2}}$ takes the following form:

$$
\begin{aligned}
& \frac{\partial u}{\partial t}=u\left(1-\frac{u}{K}\right)-\frac{u v}{\frac{u^{2}}{\alpha}+u+1}+\frac{\partial^{2} u}{\partial x^{2}} \\
& \frac{\partial v}{\partial t}=\frac{\beta u v}{\frac{u^{2}}{\alpha}+u+1}-\gamma v+D \frac{\partial^{2} v}{\partial x^{2}}
\end{aligned}
$$

Equations (5.30) describes the dynamics in the domain, $0<x<L_{x}, L_{x}=7000$. The simulation of model system 1 given in (5.30) is carried out at initial condition of the form [see 44]

$$
\begin{array}{r}
u(x, 0)=u^{*}+\epsilon\left(x-x_{1}\right)\left(x-x_{2}\right), \\
v(x, 0)=v^{*} .
\end{array}
$$

At the domain boundary, we assume no-flux boundary conditions of the form

$$
\left.\frac{\partial u}{\partial x}\right|_{(0, t)}=\left.\frac{\partial u}{\partial x}\right|_{(L, t)}=\left.\frac{\partial v}{\partial x}\right|_{(0, t)}=\left.\frac{\partial v}{\partial x}\right|_{(L, t)}=0 .
$$

In eq. (5.31), $\left(u^{*}, v^{*}\right)$ is the non-trivial equilibrium point for coexistence of the two species and $\epsilon$ is the parameter affecting the system dynamics. 
The prey and predator densities of model system 1 displays wave of chaos (WoC) phenomenon ([33], [44]) as seen from Figure 2. This WoC was originally observed and studied in detail by Petrovskii and Malchow [33]. The formation of wave patterns starts at $t=300$ and finally dominates the entire domain at $t=10000$. The dynamics does not undergo any further changes with time and remains chaotic.

a)

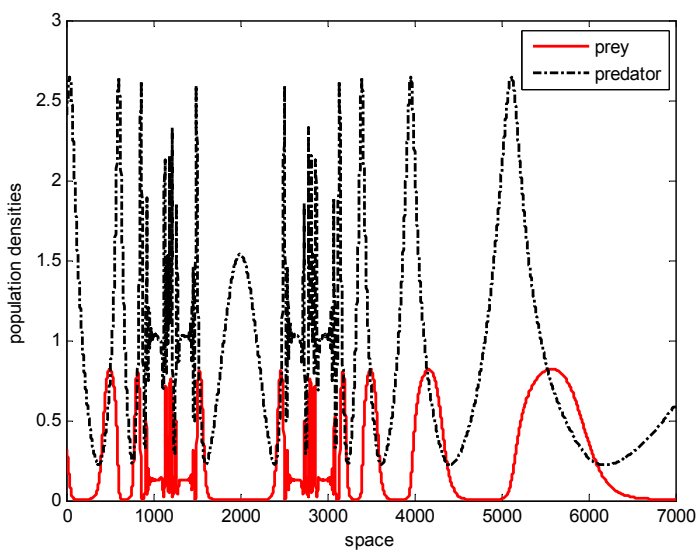

c)

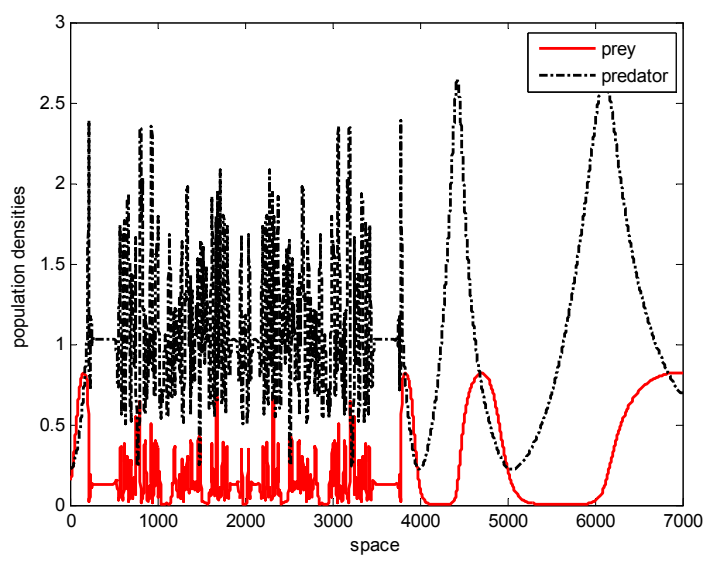

b)

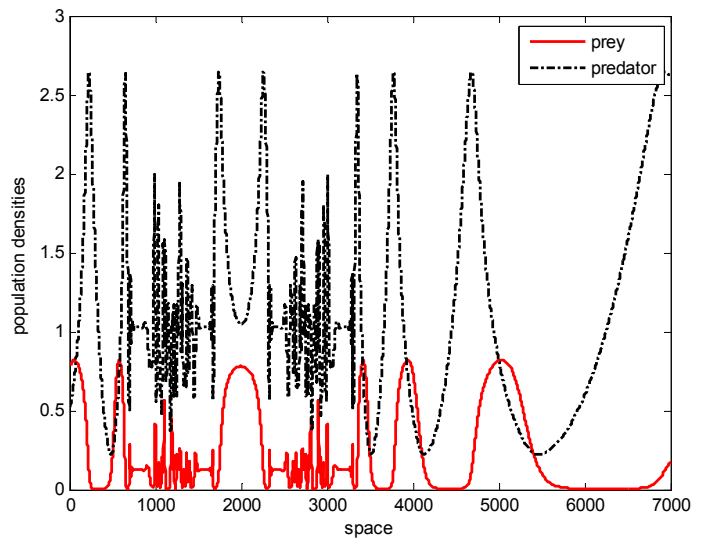

d)

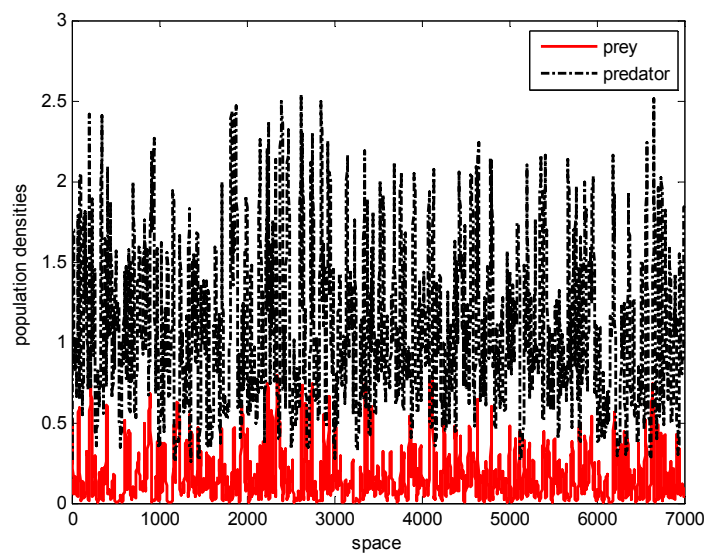

Figure 2: Model system 1 (5.30). Simulation showing route to chaos and long term persistence behaviour at (a) $t=600$ (b) $t=1000$ (c) $t=2000$ (d) $t=10000$. An extensive and long time numerical simulation is carried out for the model system. The set of parameter values at which chaotic dynamics was obtained for eqs. (5.30) with initial and boundary conditions (5.31) and (5.32) are $\alpha=0.3, \beta=2.33, \gamma=0.25, K=1, D=1$. The coexistence point is taken as $u^{*}=0.1266, v^{*}=1.0306$ with $x_{1}=1200, x_{2}=2800$ and $\epsilon=10^{-8}$. The steps $\triangle x$ and $\triangle t$ of the numerical grid are taken as 1 and 0.1 respectively. 


\subsubsection{Model system 2}

Here we study the model system 2 in one dimension. The model system (2.4) with $u(x, t), v(x, t)$ and $\Delta=\frac{\partial^{2}}{\partial x^{2}}$ takes the following form:

$$
\begin{aligned}
\frac{\partial u}{\partial t} & =u\left(1-\frac{u}{K}\right)-\frac{u v}{\frac{u^{2}}{\alpha}+u+1}+\frac{\partial^{2} u}{\partial x^{2}}, \\
\frac{\partial v}{\partial t} & =\frac{\beta u v}{\frac{u^{2}}{\alpha}+u+1}-\gamma v-\frac{f v^{2}}{v^{2}+\eta^{2}}+D \frac{\partial^{2} v}{\partial x^{2}} .
\end{aligned}
$$

a)

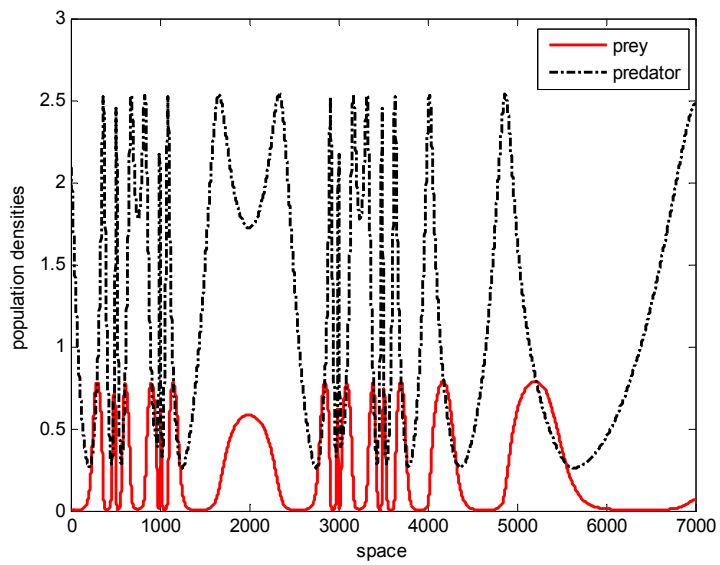

c)

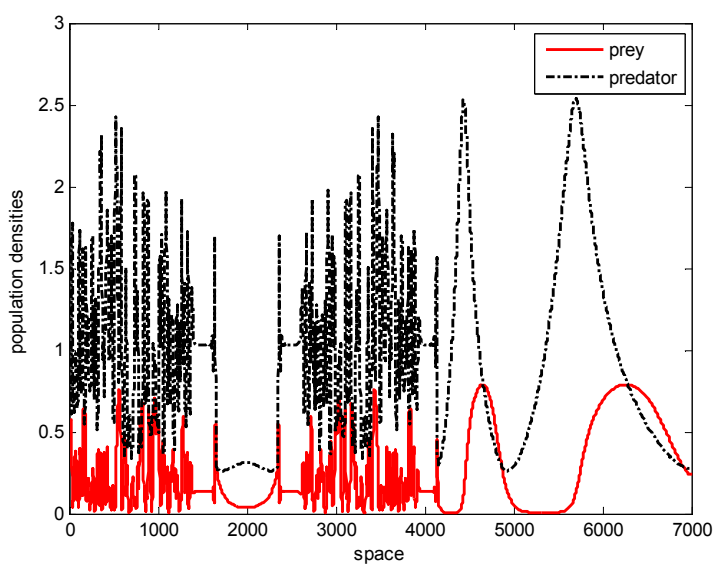

b)

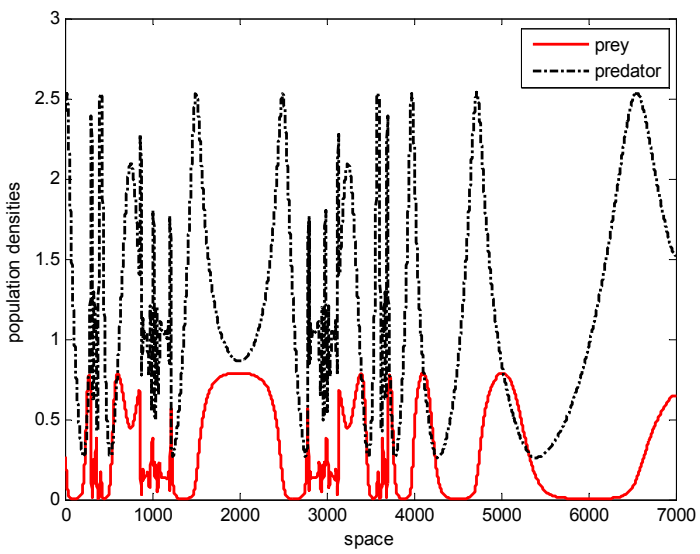

d)

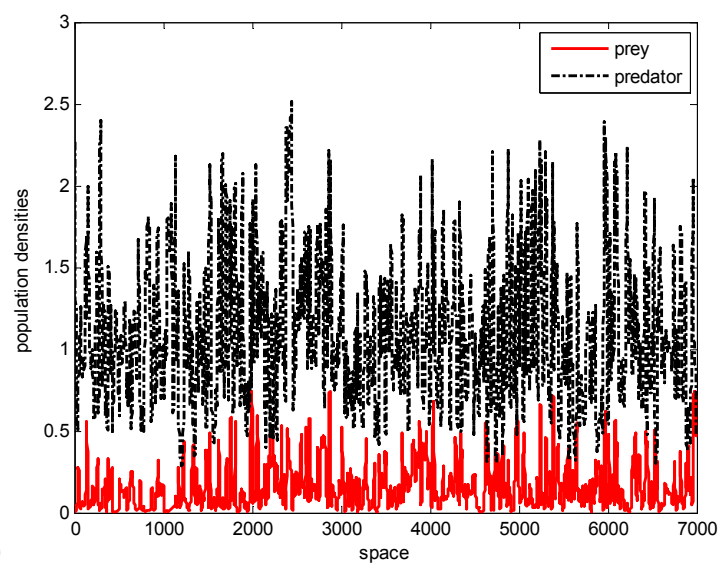

Figure 3: Model system 2, cf. (5.33). Simulation showing route to chaos and long term persistence behaviour at (a) $t=600$ (b) $t=1000$ (c) $t=2000$ (d) $t=10000$.

Numerical simulation of equations (5.33) is carried out with initial condition (5.31) and no-flux boundary condition (5.32) in the domain $0<x<L_{x}, L_{x}=7000$. For the set of parameter values $\alpha=0.3, \beta=2.33, \gamma=0.25, K=1, D=1, f=0.1, \eta=2.5$, chaotic dynamics is observed at 
$t=300$. The other parameter values considered for simulation are same as that for Figure 2 . The spatial dynamics observed for eqs. (5.33) is presented in Figure 3.

From Figure 3, we observe that the wave of chaos property persists for prey and predator species of model system 2. At different time levels, the wave of chaos travels in space and covers the whole domain at $t=10000$. If we perform a careful observation of Figures 2 and 3, we observe that although the population densities in both the model systems finally display irregular chaotic patterns, but their respective routes are remarkably different (cf. Figures (2a)-(2c) and (3a)-(3c)). This difference is very significant at time $t=2000$. The subdomain $0<x<3500$ is occupied by irregular jagged patterns for model system 1 (cf. Figure 2c) while at the same time level $t=$ 2000 model system 2 continue to display WoC phenomena where the subdomain $0<x<1500$ and $2500<x<4000$ is occupied by chaotic irregular patterns which in due course of time grows in both directions displacing the regular region $1500<x<2500$ and hence occupying the whole region (cf. Figure 3c).

\subsection{The spatiotemporal dynamics in two dimensional case}

\subsubsection{Model system 1}

In this subsection, we study the dynamics of the model system 1 in two dimensional case. Nondimensionalized system (2.3) with $u(x, y, t), v(x, y, t)$ and $\Delta=\frac{\partial^{2}}{\partial x^{2}}+\frac{\partial^{2}}{\partial y^{2}}$ can be written in the form:

$$
\begin{aligned}
& \frac{\partial u}{\partial t}=u\left(1-\frac{u}{K}\right)-\frac{u v}{\frac{u^{2}}{\alpha}+u+1}+\frac{\partial^{2} u}{\partial x^{2}}+\frac{\partial^{2} u}{\partial y^{2}} \\
& \frac{\partial v}{\partial t}=\frac{\beta u v}{\frac{u^{2}}{\alpha}+u+1}-\gamma v+D\left(\frac{\partial^{2} v}{\partial x^{2}}+\frac{\partial^{2} u}{\partial y^{2}}\right) .
\end{aligned}
$$

For the numerical simulation of model system 1, the initial distribution of the species considered is

$$
\begin{array}{r}
u(x, y, 0)=u^{*}-\epsilon_{1}(x-0.1 y-225)(x-0.1 y-675), \\
v(x, y, 0)=v^{*}-\epsilon_{2}(x-450)-\epsilon_{3}(y-150) .
\end{array}
$$

This initial condition is a two-dimensional generalization of initial condition given in eq.(5.31). At the domain boundary, zero flux boundary conditions given in eq.(5.32) is considered. The dynamics of eqs.(5.34) is considered in a rectangular domain $0<x<L_{x}$, $0<y<L_{y}$, where $L_{x}=1000, L_{y}=500$ and is effected by three parameters $\epsilon_{1}, \epsilon_{2}$ and $\epsilon_{3}$.

The numerical results obtained by semi-implicit finite difference technique for two dimensional prey-predator system are presented in Figure 4. The parameter values at which intermittent chaos was obtained for model system 1 in one dimension with initial condition (5.35) and zero flux boundary condition (5.32) are used for two dimensional analysis. Parameter values used are $\alpha=$ $0.3, \beta=2.33, \gamma=0.25, K=1, D=1, \epsilon_{1}=2 \times 10^{-7}, \epsilon_{2}=3 \times 10^{-5}, \epsilon_{3}=1.2 \times 10^{-4}$. The 
step size taken are $\triangle h=1$ and $\triangle t=1 / 3$. Spatial patterns are obtained at different time levels i.e. at $t=200,300$ and 1000 .

a)
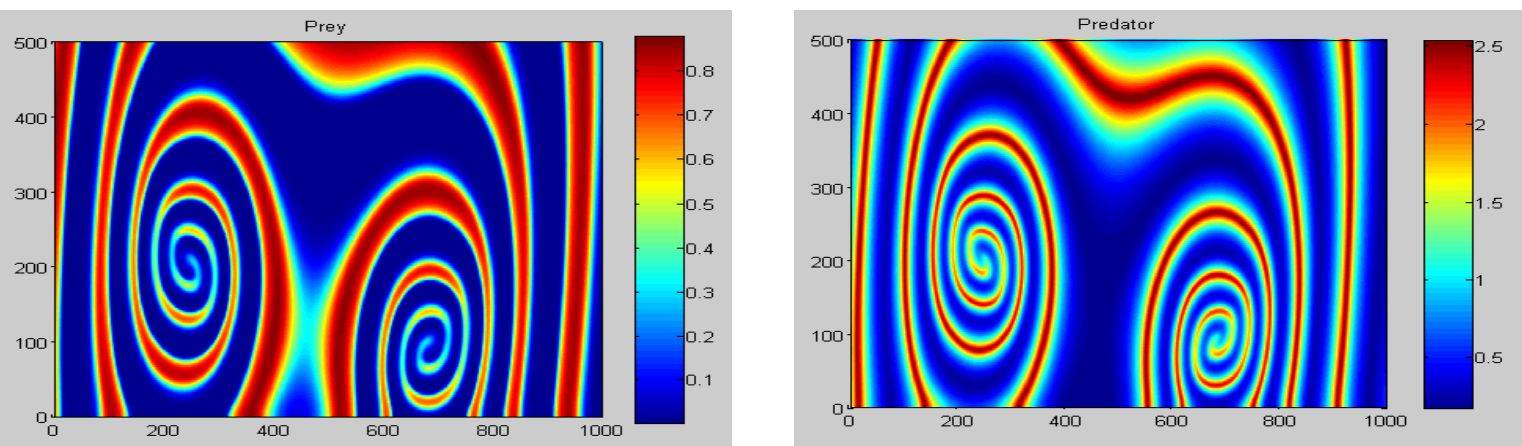

c)
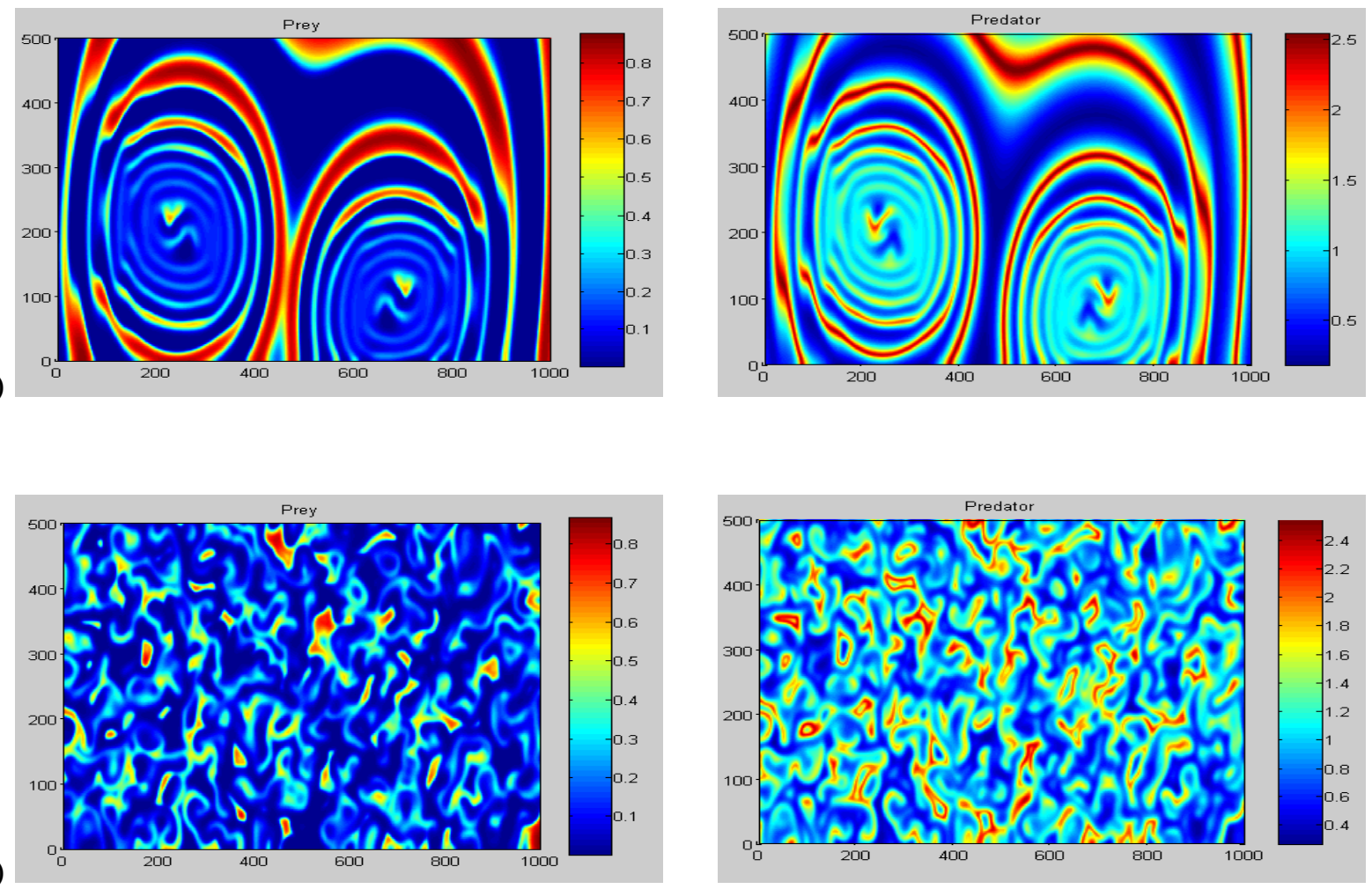

Figure 4: Spatial patterns of prey [first column] and predator [second column] densities of the model system (5.34) for (a) $t=200$, (b) $t=300$ (c) $t=1000$. Parameter values are given in the text.

The prey and predator population display regular patterns with two spiral centres which grow from their centres at $t=200$. The destruction of the two spirals begins from their centres at $t=300$, 
leading to chaotic patchy patterns at $t=1000$ (see Figure 4). This sort of spatiotemporal dynamics has been observed in similar predator-prey systems with Holling type II functional response without fish predation [28] and with fish predation [44].

\subsubsection{Model system 2}

In this subsection, the dynamics of the model system 2 is studied in two dimensional case. Nondimensionalized model system (2.4) with $u(x, y, t), v(x, y, t)$ and $\Delta=\frac{\partial^{2}}{\partial x^{2}}+\frac{\partial^{2}}{\partial y^{2}}$ can be written in the form

$$
\begin{aligned}
\frac{\partial u}{\partial t} & =u\left(1-\frac{u}{K}\right)-\frac{u v}{\frac{u^{2}}{\alpha}+u+1}+\frac{\partial^{2} u}{\partial x^{2}}+\frac{\partial^{2} u}{\partial y^{2}} \\
\frac{\partial v}{\partial t} & =\frac{\beta u v}{\frac{u^{2}}{\alpha}+u+1}-\gamma v-\frac{f v^{2}}{v^{2}+\eta^{2}}+D\left(\frac{\partial^{2} v}{\partial x^{2}}+\frac{\partial^{2} v}{\partial y^{2}}\right)
\end{aligned}
$$

The numerical simulation of model system 2 in two dimensional case is carried out with initial condition (5.35), where the values of $u^{*}$ and $v^{*}$ are obtained by solving the model system 2 without diffusion for the set of parameter values used for simulation and zero flux boundary conditions in the rectangular domain. Parameter values are fixed at $\alpha=0.3$, $\beta=2.33, \gamma=0.25, K=1, D=1, f=0.1, \eta=2.5, \epsilon_{1}=2 \times 10^{-7}, \epsilon_{2}=3 \times 10^{-5}$, $\epsilon_{3}=1.2 \times 10^{-4}$. Patterns are obtained at different time levels $t=200,300,1000$ and presented in Figure 5 .

In model system 2, formation of spiral starts at $t=200$. At $t=300$ two very closed spirals are formed for both prey and predator densities. Again we find that both the populations leads to chaotic patchy patterns at $t=1000$ which is same as that obtained for model system 1 .

\section{Discussion and Conclusion}

Modeling pattern formation in a spatial predator-prey system is a problem of immense importance for many biological and ecological applications. It has been recently shown that spaontaneous spatiotemporal pattern formation is an intrinsic property of a spatial predator-prey system $[28,33$, 35]. In this work, we have observed in simulation experiments a phenomenon of self organized chaotic patchiness $[31,32,39,40,41]$ resulting from interspecific interactions (predation and competition) and showed that although the routes are different but end patterns are the same. This suggests that fish predation has a significant role in the temporal evolution of spatial patterns of phytoplankton-zooplankton system. From a field ecologist's perspective, it is not only important to know the stable stationary patterns of an evolving phytoplankton-zooplankton $(P-Z)$ system, but also to know at what point of time these observations are carried out. For example, at $t=200$, 300, 1000 ; spatial patterns are different for the $P-Z$ system with and without fish predation. In principle, this has implications for ecological theory.

Model system 2 given in eq.(2.2) includes fish predation. The aim is to study how this activity influences pattern formation resulting from phytoplankton-zooplankton system. One should note 
a)
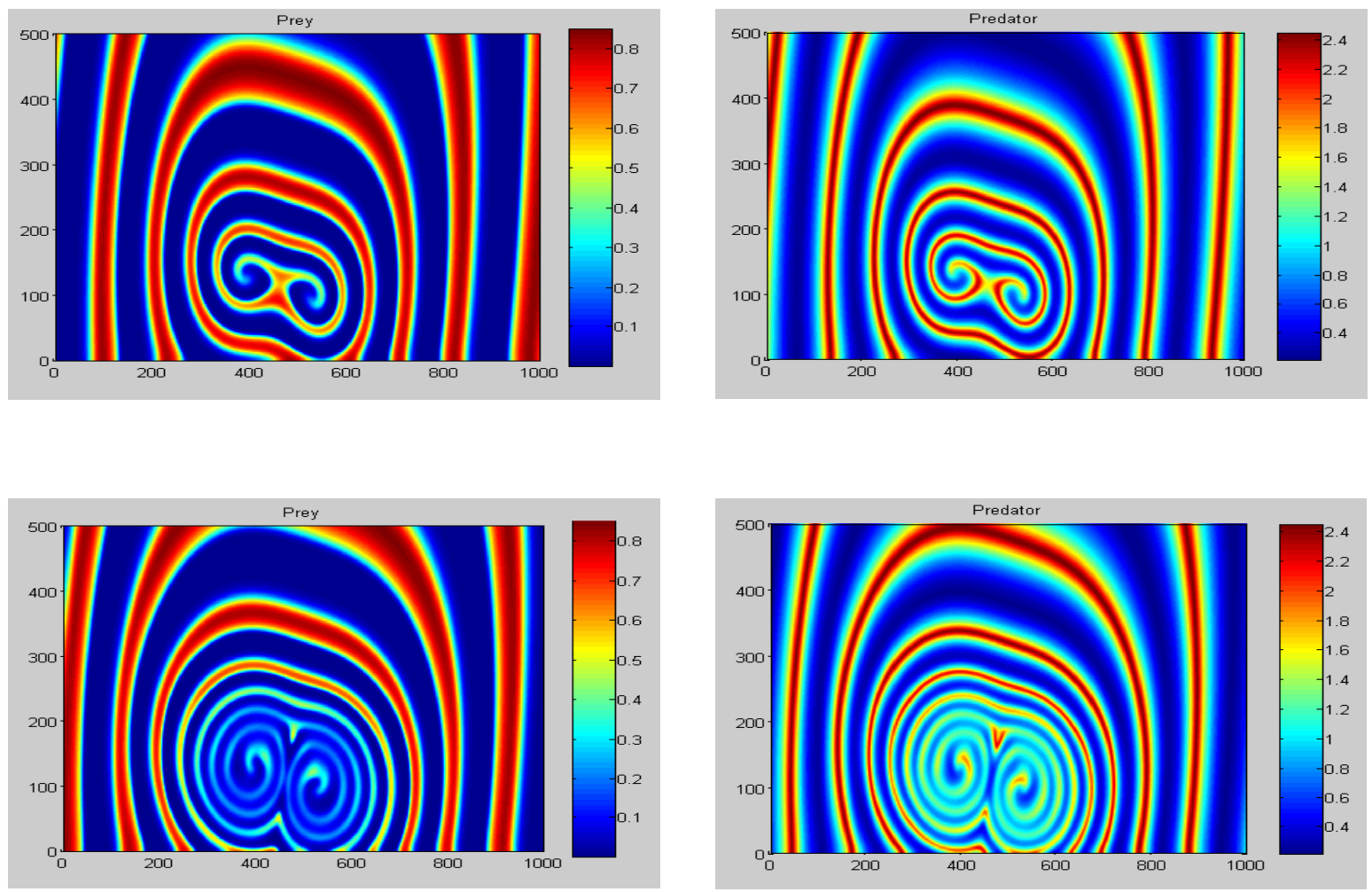

c)
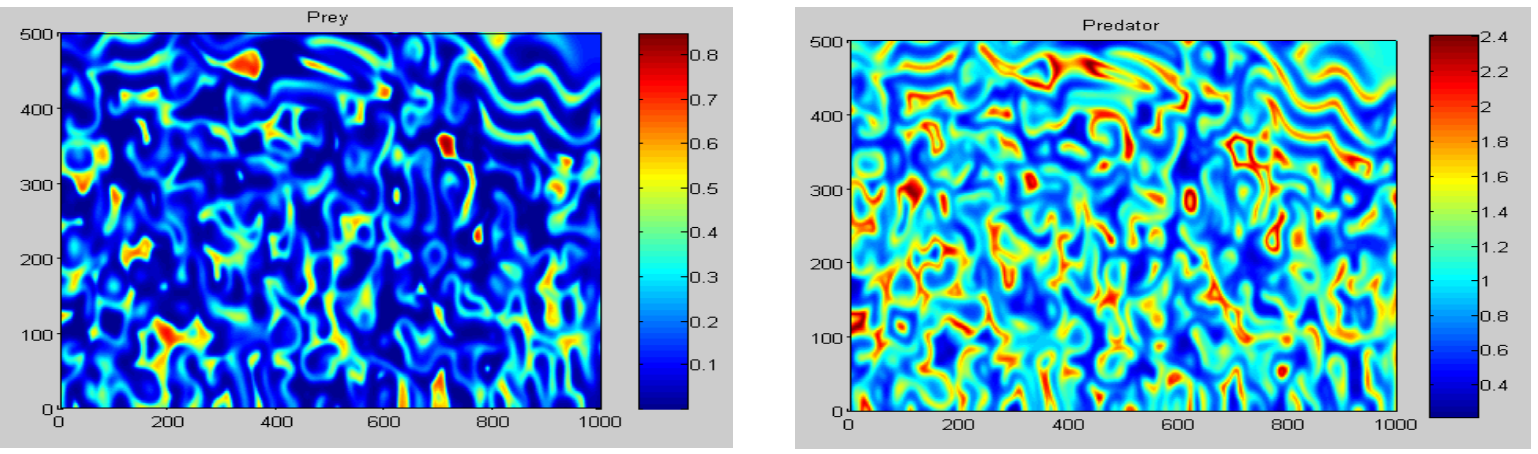

Figure 5: Spatial patterns of prey [first column] and predator [second column] densities of the model system (5.36) for (a) $t=200$, (b) $t=300$, (c) $t=1000$. Parameter values are given in the text.

that predator preference is known to alter local dynamics of plankton systems $[1,2]$. For example, phytoplankton and zooplankton are predated by fish population. As can be noted from the second R-D system (model system 2), the fish predation was considered as a fixed parameter. In marine systems, they are found cruising and feeding quite often. The present work can be extended to include behavioral traits of participating species such as fish school motion [24]. It would also be 
interesting to examine effects of positive frequency dependent predation by fishes on system on the target patterns. A target pattern is the one which represents spatial distribution of phytoplanktonzooplankton system at a given point of time (the end time of simulations). Medvinsky et al. [28] have made an important contribution in this direction.

It would be beneficial to identify mechanisms which lead to a Holling type IV functional response. For example, Bergkovist and collaborators [6] report induction of toxin production in dinoflagellates by their grazers. Toxins are poisonous chemicals. It is emanating from experiments and field studies that chemical signals can directly affect species interactions, thus, shape community structure in aquatic systems [36]. There is an urgent need to design experiments and field studies to identify $P-Z$ systems which follow such a function. These patterns of patches are generated by WoCs $[33,44]$. This should be a common mechanism as present authors have discovered them in model aquatic systems with Holling type II [44] and Holling type IV functional response functions. WoC can also be observed in a community of competing species [25, 34].

As it is well known, a continuous dissipative dynamical system with two degrees of freedom displays either oscillatory or fixed point dynamics. Since the process of pattern formation is critically dependent on local oscillatory dynamics of interacting populations, structure of its boundary with other kind of dynamical behavior (stable equilibrium point) is important. Any of these patterns can vanish if environmental noise pushes any of the parameters $\alpha, \beta, \gamma$ in the region of stable equilibrium solutions. These patterns of patches may be short-lived; the life-time would depend on time taken by the dynamics of interaction part to switch over to stable equilibrium solutions. Although these patterns of patchiness correspond to a deterministic system, these patterns will be stable only in statistical sense; the life-time would be decided by those fluctuations which are caused by transitions (stable limit cycle to fixed point solutions) of the local dynamics. Thus, from a field ecologist's perspective, it should be noted that the pace of their measurements matches the average life-time of patterns of patchiness generated in these simulation experiments.

Acknowledgements This work is supported by University Grants Commission, Govt. of India under grant no. F.33-116/2007 (SR) and Project No. UGC (32)/2008-2009/220/AM to the first author (RKU). We are thankful to Professor S. Petrovskii for having kindly drawn our attention to his works, as well as two anonymous reviewers for suggesting improvements to previous version of this manuscript.

\section{References}

[1] P. A. Abrams, H. Matsuda. Population dynamical consequences of reduced predator switching at low total prey densities. Popul. Ecol., 45 (2003), 175-185.

[2] P. A. Abrams, H. Matsuda. Consequences of behavioral dynamics for the population dynamics of predator- prey systems with switching. Popul. Ecol., 46 (2004), 13-25.

[3] J. C. Allen, C. C. Brewster, D. H. Slone. Spatially explicit ecological models: A spatial convolution approach. Chaos, Solitons and Fractals, 12 (2001), 333-347. 
[4] D. Alonso, F. Bartumeus, J. Catalan. Mutual interference between predators can give rise to Turing spatial patterns. Ecology, 83 (2002), 28-34.

[5] J. F. Andrews. A mathematical model for the continuous culture of microorganisms utilizing inhibitory substrates. Biotechnol. Bioeng., 10 (1968), 707-723.

[6] J. Bergkovist, E. Selander, H. Pavia. Induction of toxin production in dinoflagellates: the grazer makes a difference. Oecologia. Published Online, 2008.

[7] R. Bhattacharyya, B. Mukhopadhyay, M. Bandyopadhyay. Diffusion-driven stability analysis of a prey-predator system with Holling type-IV functional response. System Analysis Modelling Simulation, 43 (2003), 1085-1093.

[8] B. Boon, H. Landelout. Kinetics of nitrite oxidation by nitrobacter winogradski. Biochem. J., 85 (1962), 440 - 447.

[9] S. P. Colin, H. G. Dam. Testing for toxic effects of prey on zooplankton using sole versus mixed diets. Limnology and Oceanography, 47 (2002), 1430-1437.

[10] J.B. Collings. The effects of the functional response on the bifurcation behavior of a mite predator-prey interaction model. J. Math. Biol., 36 (1997), 149-168.

[11] V. H. Edwards. Influence of high substrate concentrations on microbial kinetics. Biotechnol. Bioeng., 12 (1970), 679-712.

[12] J. Engstrom, M. Viherluoto, M. Viitasalo. Effects of toxic and non-toxic cyanobacteria on grazing, zooplanktivory and survival of the mysis shrimp. J. Experimental and Marine Biology and Ecology, 257 (2001), 269-280.

[13] R. Ferriere, J. R. Beithoff, I. Olivierie, S. Krackow. Evolving dispersal: where to go next? TREE, 15 (2000), 5-7.

[14] H. I. Freedman, G. S. K. Wolkowicz. Predator prey systems with group defence: the paradox of enrichment revisited. Bull. Math. Biol., 48 (1986), 493-508.

[15] M. R. Garvie. Finite difference schemes for reaction-diffusion equations modeling predatorprey interactions in MATLAB. Bull. Math. Biol., 69 (2007), 931-956.

[16] J. B. S. Haldane. Enzymes. Longman, London, 1930.

[17] K. L. Kirk, J. J. Gilbert. Variation in herbivore response to chemical defenses: Zooplankton foraging on toxic cyanobacteria. Ecology, 73 (1992), 2208 - 2217.

[18] A. N. Kolmogorov. Sulla Teoria di Voltera della Lotta per I'Esisttenza. Giorn. Instituto Ital. Attuari, 7 (1936), 74-80.

[19] E. J. Koromondy. Concepts of ecology. Englewood Cliffs, Prentice Hall, New Jersey, 1969. 
[20] M. Kot. Elements of Mathematical Biology. Cambridge University Press, Cambridge, UK, 2001.

[21] S. A. Levin. The problem of pattern and scale in ecology. Ecology, 73 (1992), 1943-1967.

[22] S. A. Levin. Patchiness in marine and terrestrial systems: from individuals to populations. Proc. Trans. R. Soc. Lond. B, 343 (1994), 99-103.

[23] Q. Liu, B. Li, Z. Jin. Resonant patterns and frequency-locked induced by additive noise and periodically forced in phytoplankton-zooplankton system. Preprint: arXiv: 0705.3724, 2007.

[24] H. Malchow. Non-equilibrium spatio-temporal patterns in models of non-linear plankton dynamics. Freshwater Biology, 45 (2000), 239-251.

[25] H. Malchow, S. V. Petrovskii, E. Venturino. Spatio-temporal Patterns in Ecology and Epidemiology: Theory, Models and Simulation. CRC Press, UK, 2008.

[26] H. Malchow. Spatio-temporal pattern formation in non-linear non-equilibrium plankton dynamics. Proc. Roy. Soc. Lond. Series B, 251 (1993), 103-109.

[27] R. M. May. Stability and Complexity in Model Ecosystems. Princeton University Press, Princeton, NJ, 2001.

[28] A. Medvinsky, S. Petrovskii, I. Tikhonova, H. Malchow, B-L. Li. Spatiotemporal complexity of plankton and fish dynamics. SIAM Rev., 44 (2002), 311- 370.

[29] A. Okubo. Diffusion and ecological problems: mathematical models. Berlin, SpringerVerlag, Berlin, 1980.

[30] P. Y. H. Pang, M. X. Wang. Non-constant positive steady positive steady states of a predatorprey system with non-monotonic functional response and diffusion. Proc. Lond. Math. Soc., 88 (2004), 135-157.

[31] M. Pascual. Diffusion induced chaos in a spatial predator-prey system. Proc. Roy. Soc. London, Series B, 251 (1993), 1-7.

[32] S. V. Petrovskii, H. Malchow. A minimal model of pattern formation in a prey-predator system. Math. Comp. Modell., 29 (1999), No. 8, 49-63.

[33] S. V. Petrovskii, H. Malchow. Wave of Chaos: new mechanism of pattern formation in spatiotemporal population dynamics. Theor. Pop. Biol., 59 (2001), 157-174.

[34] S. V. Petrovskii, K. Kawasaki, F. Takasu, N. Shigesada. Diffusive waves, dynamical stabilization and spatio-temporal chaos in a community of three competitive species. Japan Journal of Industrial and Applied Mathematics, 18 (2001), 459-481.

[35] S. Petrovskii, B-L. Li, H. Malchow. Transition to spatiotemporal chaos can resolve the paradox of enrichment. Ecological Complexity, 1 (2004), 37 - 47. 
[36] G. Pohnert, M. Steinke, R. Tollrian. Chemical cues, defence metabolites and the shaping of pelagic interspecies interactions. TREE, 22 (2007), 198 - 204.

[37] V. Rai, G. Jayaraman. Is diffusion-induced chaos robust? Current Science, 84 (2003), 925 929.

[38] L. A. Segel, J. L. Jackson. Dissipative Structures: an explanation and an ecological example. J. Theor. Biol., 37 (1972), 545-559.

[39] J. A. Sherrat, B. T. Eagan, M. Lewis. Oscillations and chaos behind predator-prey invasion: Mathematical Artifact or Ecological Reality? Phil. Trans. Roy. Soc. Lond. B, 352 (1997), 21-38.

[40] J. A. Sherrat. Periodic traveling waves in cyclic predator-prey systems. Ecology Letters, 4 (2001), 30-37.

[41] J. A. Sherrat, M. Lewis, A. C. Fowler. Ecological Chaos in the wake of invasion. Proc. Natl. Acad. Sci. USA, 92 (1995), 2524-2528.

[42] A. M. Turing. On the chemical basis of morphogenesis. Philos. Trans. R. Soc. London B, 237 (1952), 37-72.

[43] R. K. Upadhyay, J. Chattopadhyay. Chaos to order: Role of toxin producing phytoplankton in aquatic systems. Nonlinear Analysis: Modelling and Control, 10 (2005), No. 4, 383-396.

[44] R. K. Upadhyay, N. Kumari, V. Rai. Wave of chaos in a diffusive system: generating realistic patterns of patchiness in plankton-fish dynamics. Chaos, Solitons and Fractals, in press.

[45] R. D. Yang, A. E. Humphrey. Dynamics and steady state studies of phenol biodegeneration in pure and mixed cultures. Biotechnol. Bioeng., 17 (1975), 1211-1235.

[46] P. Yodzis. Introduction to theoretical ecology. Harper and Row, New York, USA, 1989.

[47] L. Zhang, W. Wang, Y. Xue, Z. Jin. Complex dynamics of Holling-type IV predator-prey model. Preprint arXiv:0801.4365v1, Published online, 2008.

\section{A Kolmogorov theorem}

The Kolmogorov theorem $[18,27]$ assures the existence of either stable equilibrium point or stable limit cycle behavior in the phase space of the two dimensional (2D) dynamical systems provided nine conditions (five constraints and four requirements) are satisfied. Application of the theorem to a $2 \mathrm{D}$ system gives the constraints on parameter values which lead us to the period-doubling route to chaos. However, it is not applicable to three dimensional (3D) ODE models, which are 
commonly used to study the ecological problems.

Let a given system of equations be written as:

$$
\begin{aligned}
\frac{d H}{d t} & =H F(H, P) \\
\frac{d P}{d t} & =P G(H, P)
\end{aligned}
$$

where $H$ and $P$ are the prey and predator populations respectively at any instant of time, satisfied the conditions:

$$
\begin{array}{r}
\text { (i) } \frac{\partial F}{\partial P}<0, \text { (ii) } H\left(\frac{\partial F}{\partial H}\right)+P\left(\frac{\partial F}{\partial P}\right)<0, \text { (iii) } \frac{\partial G}{\partial P}<0, \\
\text { (iv) } H\left(\frac{\partial G}{\partial H}\right)+P\left(\frac{\partial G}{\partial P}\right)>0,(\mathrm{v}) \quad F(0,0)>0
\end{array}
$$

and fulfils the requirements

$$
\begin{array}{rll}
\text { (vi) } F(0, A)=0 \quad \text { with } \quad A>0, \quad(\text { vii }) \quad F(B, 0)=0 \quad \text { with } \quad B>0, \\
\text { (viii) } \quad G(C, 0)=0 \quad \text { with } C>0, \quad \text { (ix) } B>C,
\end{array}
$$

then the system possesses either a stable equilibrium point or a stable limit cycle, provided that $F$ and $G$ are continuous functions of $H$ and $P$ with continuous first derivatives, throughout the domain $H \geq 0, P \geq 0$.

In biological terms, Kolmogorov conditions can be defined as under [27, 46]:

Consider any given population size (as measured by numbers, biomass, etc.). Then the above conditions/requirements have the following meanings:

(i) The per capita rate of increase of the prey density is a decreasing function of the number of predators.

(ii) The rate of increase of prey density is decreasing function of both densities.

(iii) The rate of increase of predator population decreases as predator density increases.

(iv) The rate of increase of predator density is increasing function of both the densities.

(v) When both the population densities are low, the prey has positive rate of increase.

(vi) There is a predator population density sufficiently large to stop further prey growth, even when the prey is scarce.

(vii) Even in the absence of predators, the prey will not grow without bound.

(viii) The predator population, when rare, cannot grow at arbitrarily small prey densities. 
(ix) The minimum prey level that will permit an extremely sparse predator population to grow must be a level at which the prey is also capable of growth.

It is important to note that the above original Kolmogorov constraints can be sometimes relaxed such that an inequality can be replaced by an equality [27] for certain predator-prey. model. It should also be noted that these oscillations are drastically different from those found in LotkaVolterra system whose amplitude of oscillations are dependent on initial conditions. 